\title{
A matter of measurement: rotation velocities and the velocity function of dwarf galaxies
}

\author{
Chris B. Brook ${ }^{1,2 \star}$, Francesco Shankar ${ }^{3} \dagger$ \\ ${ }^{1}$ Departamento de Física Teórica, Universidad Autónoma de Madrid, 28049 Cantoblanco, Madrid, Spain \\ ${ }^{2}$ Astro-UAM, UAM, Unidad Asociada CSIC \\ ${ }^{3}$ School of Physics and Astronomy, University of Southampton, Southampton SO17 1BJ, UK
}

Accepted XXXX . Received XXXX; in original form XXXX

\begin{abstract}
The velocity function derived from large scale surveys can be compared with the predictions of $\Lambda \mathrm{CDM}$ cosmology, by matching the measured rotation velocities $\mathrm{V}_{\text {rot }}$ of galaxies to the maximum circular velocity of dark matter (DM) halos $\mathrm{V}_{\text {max }}$. For $\mathrm{V}_{\text {rot }}<50 \mathrm{kms}^{-1}$, a major discrepancy arises between the observed and $\Lambda$ CDM velocity functions. However, the manner in which different observational measures of $V_{\text {rot }}$ are associated with $V_{\max }$ is not straight forward in dwarf galaxies. We instead relate galaxies to DM halos using the empirical baryonmass to halo-mass relation, and show that different observational measures of $\mathrm{V}_{\text {rot }}$ result in very different velocity functions. We show how the W50 velocity function, i.e. using the HI profile line width at $50 \%$ of peak $\mathrm{HI}$ flux to measure $\mathrm{V}_{\text {rot }}$, can be reconciled with a $\Lambda \mathrm{CDM}$ cosmology. Our semi-empirical methodology allows us to determine the region of rotation curves that are probed by $\mathrm{HI}$ measurements $\left(\mathrm{R}_{\mathrm{HI}}\right)$, and shows that the $\mathrm{V}_{\text {rot }}$ of dwarfs are generally measured at a fraction of $R_{\max }$, explaining their tendency to have rising rotation curves. We provide fitting formulae for relating $\mathrm{R}_{\mathrm{HI}}$ and $\mathrm{R}_{\text {eff }}$ (the effective radius) to the virial radius of DM halos. To continue to use velocity functions as a probe of $\Lambda$ CDM cosmology, it is necessary to be precise about how the different measures of rotation velocity are probing the mass of the DM halos, dropping the assumption that any measure of rotational velocity can be equally used as a proxy for $\mathrm{V}_{\max }$.
\end{abstract}

Key words: galaxies: evolution - formation - haloes cosmology: theory - dark matter

\section{INTRODUCTION}

The rotation velocity $V_{\text {rot }}$ of galaxies provides a measure of the mass within the radius at which the velocity measurement was made. The velocity function of galaxies, the number density of galaxies as a function of their velocity, is thus a close kin to the mass function, the number density of galaxies as a function of mass.

The observed velocity function of galaxies has been derived by large scale HI surveys, using the HI line width as a measure of rotational velocity. The velocity (and mass) functions for dark matter halos in a $\Lambda \mathrm{CDM}$ cosmology can be readily derived for DM only simulations, with circular velocities defined as $\sqrt{G \mathrm{M}(\mathrm{r}) / \mathrm{r}}$ where $G$ is the gravitational constant, and $\mathrm{M}(\mathrm{r})$ the mass inclosed within each radius $r$. Thus, one can compare the velocity function of observed galaxies to that of dark matter halos in simulations, by

\footnotetext{
* E-mail: cbabrook@gmail.com

† E-mail: F.Shankar@soton.ac.uk
}

matching the observed rotational velocities to the circular velocity of dark matter halos. Generally, the maximum circular velocity, $\mathrm{V}_{\max }$, is chosen as representative of DM halos, although the velocity at any well defined radius could be chosen.

The velocity function derived using the half width of the HI line profile, measured at $50 \%$ of the profile peak $\mathrm{W} 50 / 2$, has been shown to differ markedly from the $\mathrm{V}_{\max }$ function expected from $\Lambda$ CDM cosmology for galaxies with W50/2 $\lesssim 50 \mathrm{~km} \mathrm{~s}^{-1}$ (Zavala et al. 2009, Zwaan et al. 2010, Trujillo-Gomez et al.|2011; Papastergis et al. 2011; Klypin et al.2014).

However, for low mass galaxies, the relation between $\mathrm{W} 50 / 2$, and $\mathrm{V}_{\max }$ becomes less clear than for more massive disc galaxies for several reasons: low mass galaxies have a higher ratio of velocity dispersion to rotation velocity, are thicker, with less regular HI discs, rising rotation curves which have not reached a maximum, and decreasing baryon fractions meaning that baryons may be confined to increasingly central regions of the DM halo.

To make a more accurate comparison between observed and predicted velocities distributions Papastergis et al. (2015) relaxed the assumption that $\mathrm{W} 50 / 2$ and $\mathrm{V}_{\max }$ can be directly compared, and instead matched W50/2 velocities to the velocity of DM halos 


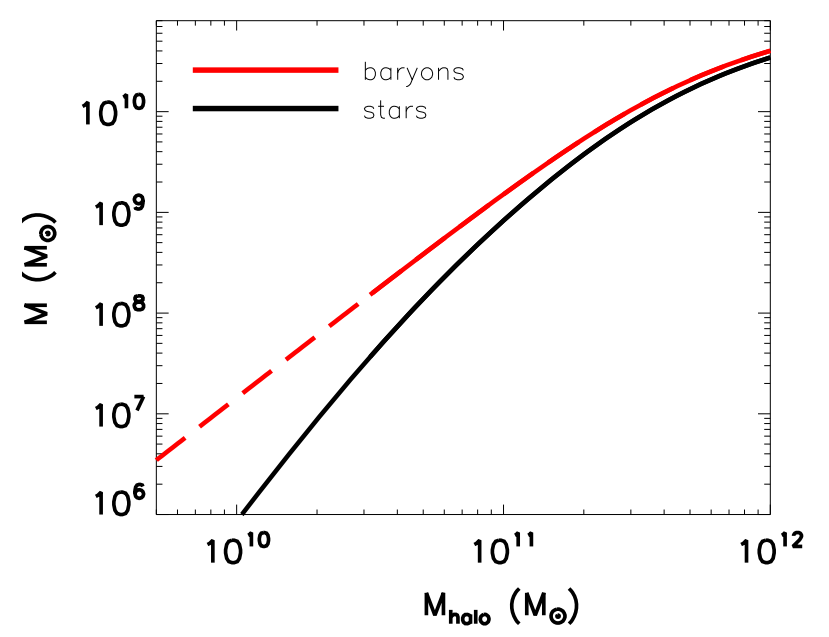

Figure 1. The abundance matching relation adopted in this study, $\mathrm{M}_{\mathrm{b}}$ $\mathrm{M}_{\text {halo }}$ (red line) and $\mathrm{M}_{*}-\mathrm{M}_{\text {halo }}$ (black line). The region that is extrapolated from the Papastergis $2012 \mathrm{M}_{\mathrm{b}}-\mathrm{M}_{\text {halo }}$ relation is dashed.

at the largest radius to which the observed $\mathrm{HI}$ gas extends $\mathrm{R}_{\text {last }}$. The mismatch between theory and observation was shown to persist, although this procedure has its own issues, such as the assumption of an NFW density profile for the DM halos, the assumption that concentration of the galaxies' halos match those of pure N-body simulations, and the assumption that $\mathrm{W} 50 / 2$ reflects the rotational velocity of dwarf galaxies at $\mathrm{R}_{\text {last }}$.

In this study, we put aside the issue of how to best match observed and theoretical velocities, and instead match observed galaxies to DM halos based on mass. Galaxies with regular, extended HI discs and well studied rotation curves, have been shown to follow a very tight relation between baryonic mass and circular velocity, the Baryonic Tully Fisher relation (BTFR). The small scatter in this relation provides compelling evidence that the baryonic mass of galaxies is tightly correlated with their total mass (e.g. McGaugh 2005). We propose that matching baryonic mass to halo mass provides a more reliable mapping between observed galaxies and DM halos, than does the mapping between velocities. Several studies have explored the relation between galaxy masses, and the mass of DM halos (e.g. Shankar et al. 2006, Moster et al. 2010, Guo et al. 2010. Papastergis et al. 2012)

Part of our motivation comes from the fact that the tight relation between baryon mass and rotation velocity when measured from the flat part of the rotation curve is not well reproduced, in terms of scatter, when one looks at the HI line-widths of low mass galaxies. For example, Trachternach et al. (2009) explored 11 dwarf galaxies with baryonic masses $0.14<\mathrm{M}_{\mathrm{b}}<12.92 \times 10^{8} \mathrm{M}_{\odot}$, and find that 6 have well defined rotation curves and do fit on the BTFR, while 5 others have W50/2 values that fall well below the BTFR: these 5 galaxies also have poorly defined rotation curves. When matching observations to DM halos by velocities, these latter 5 galaxies would be placed in low mass halos. The study of 101 dwarf galaxies in Geha et al. (2006), selected as low luminosity SDSS galaxies, also shows a large scatter in the values of W20 in a sample of galaxies with small range of baryon masses.

The other point worth considering is that matching W50/2 to $\mathrm{V}_{\text {max }}$ leads to many dwarf galaxies being matched to low mass halos, implying baryon fractions that are higher than what is found in $\mathrm{L}^{*}$ galaxies. For example galaxies with $\mathrm{W} 50 / 2 \sim 10 \mathrm{~km} \mathrm{~s}^{-1}$, when assuming this reflects $\mathrm{V}_{\max }$, are matched to halos with

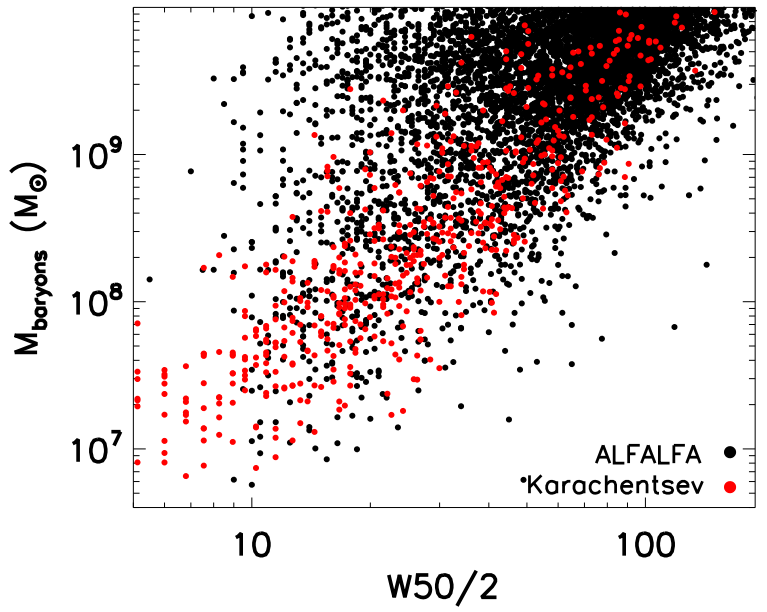

Figure 2. The baryon mass versus W50/2 for ALFALFA galaxies (black dots) and the Local Volume catalogue of Karachentsev 2013 (red dots). These data sets share many galaxies. The data have similar distributions of low mass galaxies where the Local Volume statistics are better.

$\mathrm{M}_{\text {halo }} \sim 3 \times 10^{8} \mathrm{M}_{\odot}$ (Figure 10 in Klypin et al. 2014), even though they have $\mathrm{M}_{\mathrm{b}} \sim 10^{7} \mathrm{M}_{\odot}$.

Our contention is that the low value of W50/2 in many dwarfs is probing relatively inner regions of rising rotation curves, and therefore the $\mathrm{M}_{\mathrm{b}}-\mathrm{M}_{\text {halo }}$ relation is a more robust manner for connecting galaxies to DM halos. We will see in particular that different measures of $V_{\text {rot }}$ result in very different velocity functions, meaning that they cannot all be matched to $V_{\max }$ equally.

Matching galaxies to halos using mass provides an interesting, and we believe, compelling way to compare observed and theoretical velocity functions. The study is organised as follows: in section 2 we outline our procedure, then in section 3 we show the adopted empirical relation between $\mathrm{M}_{\mathrm{b}}$ and $\mathrm{M}_{\text {halo }}$ and then show how $\mathrm{M}_{\mathrm{b}}$ is related to $\mathrm{V}_{\text {rot }}$ in various observational data sets using various measures of $\mathrm{V}_{\text {rot }}$. We then combine these relations, and show how differences in the $\mathrm{M}_{\mathrm{b}}-\mathrm{V}_{\text {rot }}$ relation affect the derived velocity function. We conclude that $\Lambda \mathrm{CDM}$ is compatible with the velocity function observations, under the condition that it can explain the different BTFRs. In an appendix, we show that our results are robust to different data sets.

\section{METHODS}

Our procedure for deriving the velocity function is as follows:

- use an empirical abundance matching between $\mathrm{M}_{\text {halo }}$ and $\mathrm{M}_{\mathrm{b}}$.

- use observed data sets to relate $\mathrm{M}_{\mathrm{b}}$ to $\mathrm{V}_{\text {rot }}$ where the relation is dependent on the specific manner in which rotation velocity is measured (using HI line widths or rotation curves).

- for each $\mathrm{M}_{\text {halo }}$ we thus have empirically matched $\mathrm{M}_{\mathrm{b}}$ and $\mathrm{V}_{\text {rot }}$.

- we then use the mass function from N-body simulations which gives us the predicted number of halos of each mass, and hence the predicted number of galaxies with particular values of $\mathrm{M}_{\mathrm{b}}$ and $\mathrm{V}_{\text {rot }}$, the latter being the velocity function.

In our procedure, galaxies have been matched to halos according to their mass. We have not attempted to separate satellites and central galaxies which will be explored in a subsequent study; for the theoretical mass function we simply use a power law fit to the simulations Klypin et al. (2011 2014). With galaxies matched to ha- 


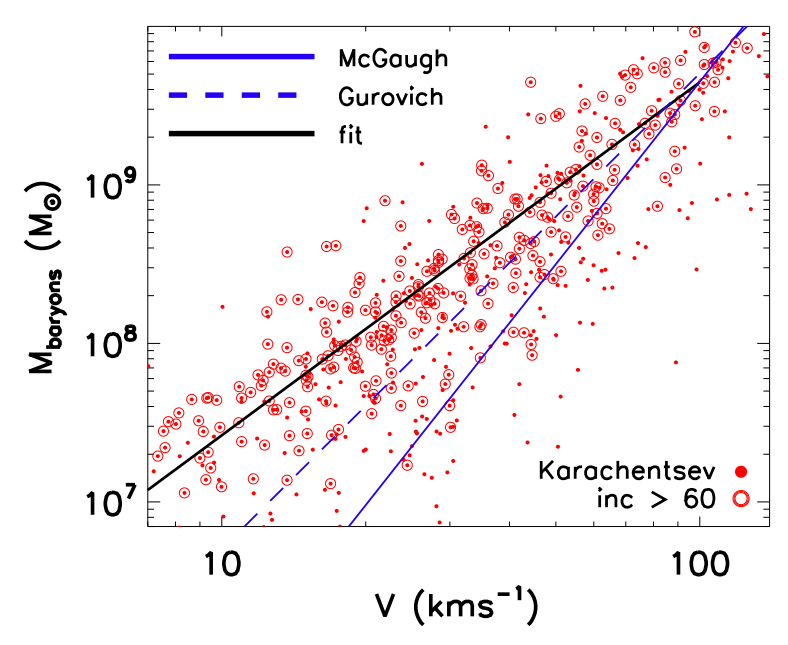

Figure 3. The baryonic Tully-Fisher relation (BTFR) for the Karachentsev 2013 galaxies, with a fit to galaxies which have inclinations inc $>60^{\circ}$. We use $\mathrm{V}_{50}$, the W50 line half widths adjusted for inclination and velocity dispersion. Also shown are the BTFRs of McGaugh 2012, derived using $\mathrm{V}_{\text {flat }}$ and from Gurovich 2010, derived using W20.

los using the adopted $\mathrm{M}_{\mathrm{halo}}$ and $\mathrm{M}_{\mathrm{b}}$ relation, the resultant velocity function differs depending on the adopted relation between $\mathrm{M}_{\mathrm{b}}$ to $V_{\text {rot }}$ i.e the BTFR, which itself differs according to the manner in which $\mathrm{V}_{\text {rot }}$ is measured.

For our purposes, we are interested in adopting a single relation between each of the galaxy properties $\mathbf{M}_{\text {halo }}, \mathrm{M}_{*}$ and $\mathbf{M}_{\text {gas }}$ and then exploring the effect of convolving these relations with different BTFRs. In the appendix we start with a different set of relations between $M_{\text {halo }}, M_{*}$ and $M_{\text {gas }}$ based on the recent study of Bradford et al. (2015), and then repeat our procedure. The results are very similar to those presented in the paper, indicating that our results are robust. Within the paper, we also make several other consistency checks between different data sets. We neglect scatter in our adopted scaling relations as it will not affect our final determination of the low-velocity end of the velocity function.

\section{ANALYSIS}

\section{1 $\mathbf{M}_{\mathrm{b}}-\mathbf{M}_{\text {halo }}$}

We take a fit to the $\mathrm{M}_{\mathrm{b}}-\mathrm{M}_{\text {halo }}$ relation from Papastergis et al. (2012), using the equation:

$$
\mathrm{M}_{\mathrm{b}}=0.0635 \mathrm{M}_{\text {halo }}\left[\left(\frac{\mathrm{M}_{\text {halo }}}{10^{11.65}}\right)^{-1.057}+\left(\frac{\mathrm{M}_{\text {halo }}}{10^{11.65}}\right)^{0.556}\right]^{-1}
$$

This has the same functional form as the $\mathrm{M}_{*}-\mathrm{M}_{\text {halo }}$ relation as Moster et al. (2010). The relation is shown as a red line in Figure 1, with the region that is extrapolated from the Papastergis et al. (2012) relation shown as a dashed line. We then compute the gas and stellar composition of the baryons using equation 1 of Papastergis 2012,

$$
\log _{10}\left(\mathrm{M}_{\mathrm{HI}} / \mathrm{M}_{*}\right)=-0.43 \log _{10}\left(\mathrm{M}_{*}\right)+3.75
$$

The adopted relation between gas and stellar mass of galaxies is also consistent with the empirical relation derived by Peeples \& Shankar (2011). The residual $\mathbf{M}_{*}-\mathbf{M}_{\text {halo }}$ relation, shown as a black

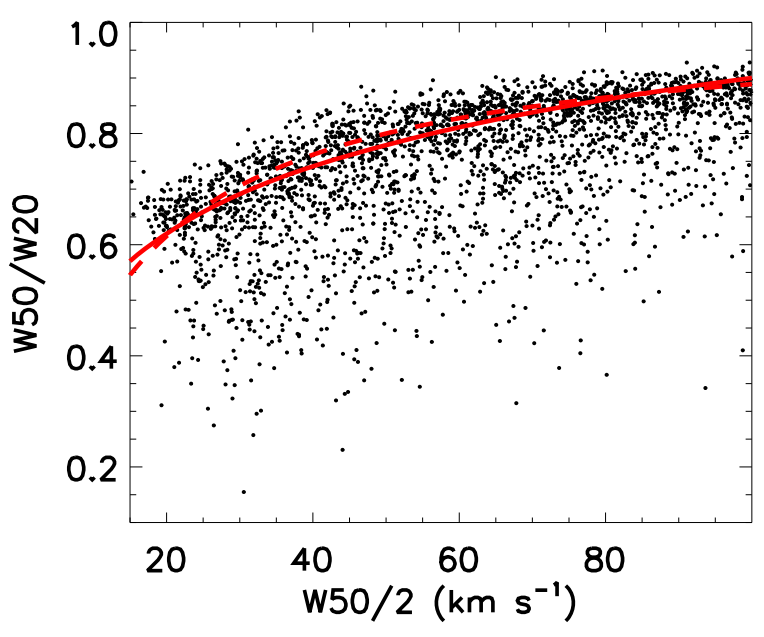

Figure 4. HIPASS data showing the difference between HI line width measured at $20 \%$ and $50 \%$ of the peak flux. We plot W50/2 versus W50/W20. For low mass galaxies, there is a big difference in the two indicators of rotation velocity, with a large tail toward low values of W50/W20. The fitted red line will be used to show the change in the velocity function when using W20, as compared to W50, as an indicator of rotation velocity. The red dashed line shows the result of using $\mathrm{W} 20=\mathrm{W} 50+25 \mathrm{~km} \mathrm{~s}^{-1}$.

line in Figure 1, is close to the Guo et al. (2010) relation. It has been shown that the extrapolation of Guo et al. (2010) to lower mass galaxies gives a good approximation of abundance matching results for Local Group galaxies (Brook et al.2014).

Galaxies are matched down to halo masses of around $7 \times 10^{9}$. Below this limit, several studies have shown that galaxy formation is suppressed by the early re-ionisation of the intergalactic medium (Bullock et al. 2000, Somerville 2002, Benson et al. 2002).

\section{2 $\mathrm{M}_{\mathrm{b}}-\mathrm{V}_{\text {rot }}$ : measures of the BTFR}

We first explore the relation between W50/2 and baryonic mass for ALFALFA galaxies (Haynes et al. 2011), and for the Local Volume (LV) galaxies as compiled by Karachentsev et al. (2013). We calculate $\mathrm{M}_{*}$ for the ALFALFA galaxies following Bell et al. (2003) and calculate $\mathrm{HI}$ mass $\mathrm{M}_{\mathrm{HI}}=2.36 \mathrm{e} 5 \mathrm{~d}^{2} \times$ flux, where flux is the $\mathrm{HI}$ flux and $d$ is the distance in $\mathrm{Mpc}$, then $\mathrm{M}_{\mathrm{b}}=\mathrm{M}_{*}+4 / 3 \mathrm{M}_{\mathrm{HI}}$. We plot, in Figure 2, $\mathrm{M}_{\mathrm{b}}$ versus W50/2, where the ALFALFA galaxies are shown as black circles and the LV galaxies as red circles. The distributions are similar for the low velocity region we are interested in, and in fact there is significant overlap in the data sets.

We define

$$
\mathrm{V}_{50}=\sqrt{(\mathrm{W} 50 / 2 / \sin (\mathrm{inc}))^{2}-8^{2}}
$$

taking account of inclination and velocity dispersion in deriving a measure of rotation velocity. In Figure 3 we then take the LV galaxies and plot the $\mathrm{V}_{50}$ BTFR (red dots) and use galaxies that have inclinations inc $>60^{\circ}$ (red circles) to make a fit (black line):

$$
\log _{10} \mathrm{M}_{\mathrm{b}}=5.19+2.22 \log _{10} \mathrm{~V}_{50}
$$

Also shown in Figure 3 are two BTFRs taken from the literature; the BTFR derived using the flat part of the rotation curve $V_{\text {flat }}$ of galaxies with regular, extended $\mathrm{HI}$ discs and well resolved rotation curves, $\log _{10} \mathrm{M}_{\mathrm{b}}=2.01+3.82 \log _{10} \mathrm{~V}_{\text {flat }}$ (McGaugh 2012); and the BTFR derived using inclination and dispersion adjusted W20 measurements, $\log _{10} \mathrm{M}_{\mathrm{b}}=3.7+3 \log _{10} \mathrm{~V}_{20}$ (Gurovich et al. 2010). 


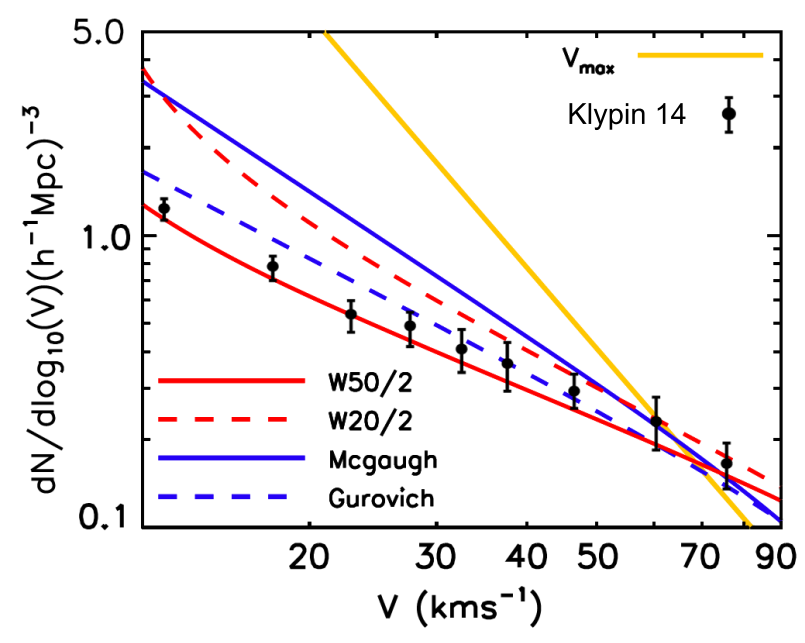

Figure 5. The yellow line shows the $\mathrm{V}_{\max }$ velocity function of DM only simulations. We then show the velocity function predicted by $\Lambda \mathrm{CDM}$ cosmology when making different assumptions about the BTFR, having matched observed baryon masses to DM halo masses. Using the W50/2 BTFR, equation 4 is shown as the red line. The W20/2 BTFR, derived using equation 5 is shown as a red dashed line. Results of assuming the BTFRs of Mcgaugh (2012) and Gurovich et al. (2010) are shown as blue solid and blue dashed lines. The observational results from Klypin et al. (2014) are shown as black circles with error bars

\subsection{W50 compared to W20}

Here, we highlight the difference between W50 and W20, in low mass galaxies, i.e. the difference between measuring the HI linewidths at $20 \%$ and $50 \%$ of the peak value. We use data taken from the HOPCAT catalogue (Doyle et al.2005) of the HIPASS survey, but also show the relation from the recent study of Bradford et al. (2015), using the ALFALFA catalogue.

Figure 4 shows the ratio W50/W20 as a function of W50/2. For low mass galaxies, there is a significant difference in the two indicators of rotation velocity, with a large tail toward low values of W50/W20. We will use the fitted red line,

$$
\mathrm{W} 50 / \mathrm{W} 20=0.1+0.4 \log _{10}(\mathrm{~W} 50 / 2)
$$

to adjust our velocity function from one based on W50, to one based on W20, shown as dashed red lines in figures $4,6 \& 7$, highlighting the effect of choosing W50 over W20 as an indicator of rotation velocity. This adjustment is almost identical to simply using W20=W $50+25 \mathrm{~km} \mathrm{~s}^{-1}$ (dashed red line in Figure 4), which is the typical difference between these measures of $\mathrm{V}_{\text {rot }}$ according to both Bradford et al. (2015) and Koribalski et al. (2004).

\subsection{The Velocity Function}

In Figure 5 we explore the effect of different BTFRs on the velocity function, when the BTFRs are used with our adopted $\mathrm{M}_{\mathrm{b}}-\mathrm{M}_{\text {halo }}$ relation. In each case $V_{\text {rot }}$ values are adjusted to line of sight velocities $\mathrm{V}_{\text {los }}$ by averaging over all inclinations to allow direct comparison with the observed W50/2 velocity function ${ }^{1}$ Klypin et al. 2014, Papastergis et al. 2015).

1 We have not included a dispersion correction as the observed velocity functions use W50/2 directly. We derived the $\mathrm{V}_{50}$ BTFR having used an inclination and dispersion corrected W50/2, because such relation follows a single power law, with lower scatter, than the W50/2 BTFR. The re-inclusion of the velocity dispersion when deriving the velocity function

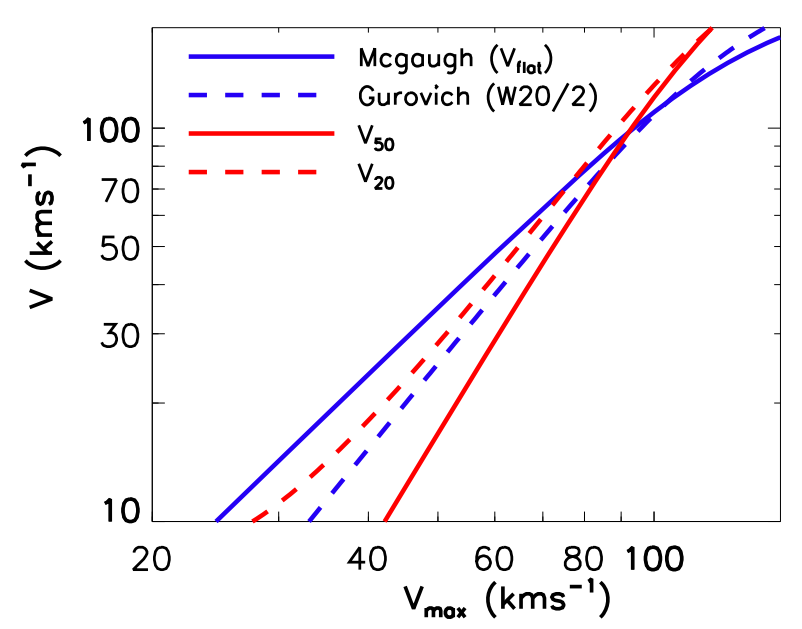

Figure 6. We plot $\mathrm{V}_{\text {rot }}$ as a function of the $\mathrm{V}_{\max }$ of the matched halo, under different assumptions for the BTFR after matching galaxies to halos on the basis of mass. We use 2 different empirical BTFRs from the literature, shown as blue lines. We also use the fit to $\mathrm{W}_{50}$ from figure 2 (red line) and the adjustment from W50 to W20 as shown in Figure 4 (red dashed line).

The yellow line in Figure 5 , shows the $\mathrm{V}_{\max }$ velocity function of DM only simulations, while the the velocity function derived from the W50/2 BTFR, equation 4 , is shown as the red line. The velocity function derived from the W20/2 BTFR using equations 4\& 5 is shown as a red dashed line. Results of assuming the BTFRs of McGaugh (2012), and Gurovich et al. (2010) are shown as blue solid and blue dashed lines respectively. The effect of different BTFRs on the velocity function is significant.

Consider the difference between the velocity function derived using the $\mathrm{V}_{\text {flat }} \mathrm{BTFR}$, i.e. the blue solid line, as compared to the yellow line which shows the $V_{\max }$ velocity function predicted from pure N-body simulations. The difference is large, yet hydrodynamical galaxy formation simulations within a $\Lambda \mathrm{CDM}$ cosmological context have been shown to simultaneously match both the $\mathrm{M}_{\mathrm{b}}$ $\mathrm{M}_{\text {halo }}$ and the $\mathrm{V}_{\text {flat }}$ BTFR relation (Santos-Santos et al. submitted), which is sufficient to reconcile the lines.

The red line, derived using the $\mathrm{V}_{50}$ BTFR, shows the W50/2 velocity function, which is very similar to the observed W50/2 velocity function (e.g. Klypin et al.|2014, shown as black cycles with error bars in Figure 5.

\subsection{Relating various measures of $V_{\text {rot }}$ to $V_{\max }$}

Having matched galaxies to DM halos based on mass, we can explore the implied links between various measures of $V_{\text {rot }}$ for the observed galaxies, and the $\mathrm{V}_{\max }$ of matched DM halos. This is shown in Figure 6 which shows the results for the different BTFRs. The $V_{\text {rot }}$ taken from the two empirical BTFRs, as seen in earlier plots, are shown as a function of the $\mathrm{V}_{\max }$ of the matched DM halos (blue lines). Results using the $\mathrm{V}_{50}$ BTFR, i.e. equation 4 are shown as the solid red line, and the adjustment from W50 to W20, equation 5 as the red dashed line.

is what causes the upturn in the W50/2 or W20/2 velocity functions at low velocities. The final result will not change if the velocity dispersion is maintained throughout, so long as one selects an appropriate (but more complicated) relation between baryon mass and measured W50/2. 


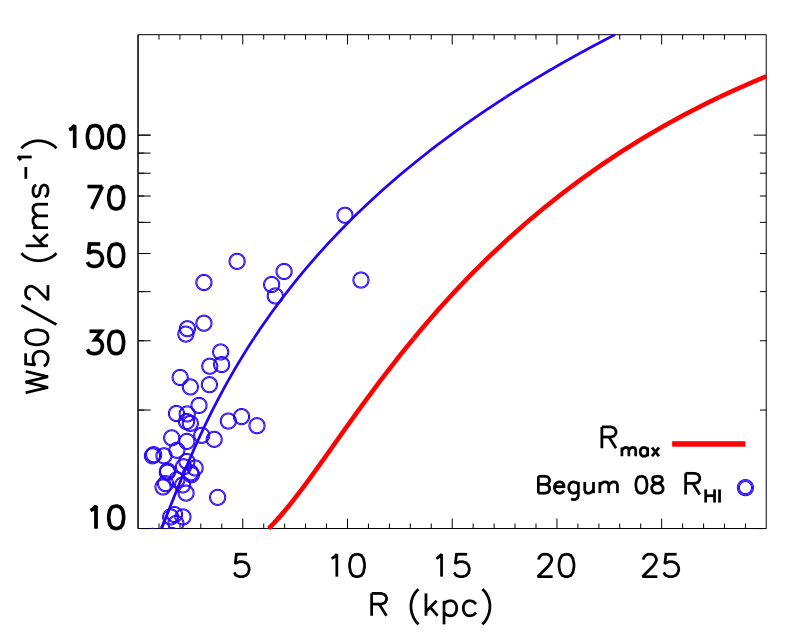

Figure 7. The red line is the $\mathrm{R}_{\max }$ of $\mathrm{DM}$ halos, plotted against the value of W50/2 for the matched galaxies. The blue circles show the radial extent of the HI discs $\left(\mathrm{R}_{\mathrm{HI}}\right)$ versus W50/2 of the FIGGs (Begum et al. 2008) sample. The blue line takes the fit of $\mathrm{R}_{\mathrm{HI}}$ versus HI gas mass from Begum (2008), and converts from $\mathrm{HI}$ mass to W50/2 using equations $2 \& 4$

\subsection{The extent of low mass galaxies}

Finally, we highlight the difference between the extent of HI gas in galaxies, and the $R_{\max }$ of their matched DM halos, where $R_{\max }$ is the radius at which $V_{\max }$ is reached. In Figure 7. W50/2 is plotted as a function of $\mathrm{R}_{\mathrm{HI}}$, defined as the radius at which the $\mathrm{HI}$ gas density reached 19 atoms $\mathrm{cm}^{-2}$, for the FIGGs sample of galaxies (Begum et al.2008) shown as blue circles, a representative subset of the compiled Karachentsev et al. (2013) data.

The red line in Figure 7 shows the $\mathrm{R}_{\max }$ of DM halos, plotted against the value of W50/2 for the matched galaxies. The blue circles show the radial extent of the $\mathrm{HI}$ discs $\left(\mathrm{R}_{\mathrm{HI}}\right)$ versus W50/2 for the FIGGs sample.

The blue line in Figure 7 uses equation 1 of Begum et al. (2008) which relates $\mathrm{HI}$ mass to $\mathrm{R}_{\mathrm{HI}}$, along with our equations $2 \& 4$ This is a good consistency check for the data sets.

In Figure 8, we plot the ratios $\mathrm{R}_{\mathrm{HI}} / \mathrm{R}_{\mathrm{vir}}$ and $\mathrm{R}_{\mathrm{eff}} / \mathrm{R}_{\mathrm{vir}}$ for observed galaxies and their matched halos. $\mathrm{R}_{\mathrm{HI}}$ comes from the FIGGS sample Begum (2008) while $\mathrm{R}_{\text {eff }}$ from the sample of Bradford et al. 2015. The black line and grey area at $0.015 \mp 0.005 \times$ $\mathrm{R}_{200}$, is the relation found by Kravtsov (2013) for $\mathrm{R}_{\mathrm{eff}}$ of more massive galaxies. A constant ratio of $\mathrm{R}_{\text {eff }} / \mathrm{R}_{\mathrm{vir}}$ does not hold for lower mass galaxies, which are confined to more central regions within their host halos. The ratios are well fit power laws

$$
\log _{10} R_{\mathrm{HI}} / \mathrm{R}_{\mathrm{vir}}=-7.65+0.6 \log _{10} \mathrm{M}_{\text {halo }}
$$

and

$$
\log _{10} R_{\text {eff }} / R_{\text {vir }}=-7.25+0.49 \log _{10} \mathrm{M}_{\text {halo }}
$$

\section{DISCUSSION}

When matching W50/2 velocities of observed galaxies to $\mathrm{V}_{\max }$ of DM halos, one finds a significant excess in the number of DM halos with $\mathrm{V}_{\max } \lesssim 50 \mathrm{~km} \mathrm{~s}^{-1}$. This mismatch occurs where halos are too massive to have had galaxy formation prevented by re-ionisation; from this perspective the observed velocity function indicates that there is a "too big to fail" problem (Zavala et al. 2009, Klypin et al. 2014. Papastergis et al. 2015).

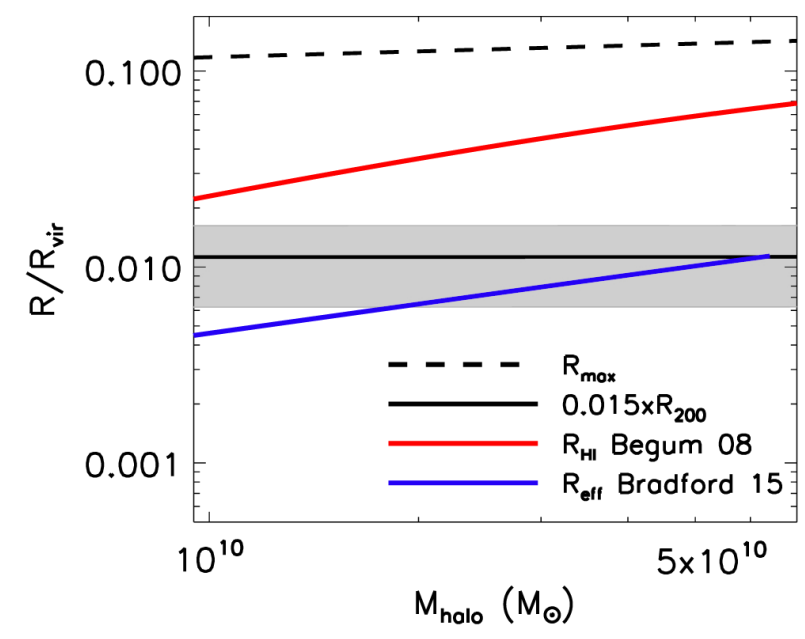

Figure 8. The ratios $\mathrm{R}_{\mathrm{HI}} / \mathrm{R}_{\mathrm{vir}}$ and $\mathrm{R}_{\mathrm{eff}} / \mathrm{R}_{\mathrm{vir}}$ for observed galaxies and their matched halos. $\mathrm{R}_{\mathrm{HI}}$ comes from Begum (2008) while $\mathrm{R}_{\text {eff }}$ from Bradford et al. 2015. The black line and grey area at $0.01 \mp 0.005 \times R_{200}$, is the relation found by Kravtsov (2013) for $\mathrm{R}_{\text {eff }}$ of more massive galaxies.

In this study we instead match observed galaxies to DM halos based on baryonic mass, and explored the impact of the various forms of the BTFR on the velocity function that is expected in $\Lambda \mathrm{CDM}$ cosmology. The BTFR derived from $\mathrm{W} 50 / 2$ values taken from large surveys of galaxies, results in a very different prediction for the velocity function than the one inferred from the BTFR derived using the $\mathrm{V}_{\text {flat }}$ values of dwarf galaxies that have extended, regular HI rotation fields. The question of whether the observed velocity function is consistent with $\Lambda \mathrm{CDM}$ cosmology may be reduced to the question of whether the various forms of the BTFR can be reproduced within a $\Lambda \mathrm{CDM}$ cosmology.

Yet it is has already been shown that hydrodynamical cosmological galaxy formation simulations are able to simultaneously match the $\mathrm{V}_{\text {flat }}$ BTFR and $\mathrm{M}_{\mathrm{b}}-\mathrm{M}_{\text {halo }}$ relation (Santos-Santos et al. 2015 submitted, see also Brook et al. 2012, Aumer et al. 2013, which is sufficient to reconcile the observed $V_{\text {flat }}$ velocity function with $\Lambda \mathrm{CDM}$ predictions. Therefore, when considering galaxy formation processes, the predictions of $\Lambda \mathrm{CDM}$ are not embodied by the yellow line that is derived from purely DM only simulations: simulated galaxies within a $\Lambda \mathrm{CDM}$ context are compatible with the observed $\mathrm{V}_{\text {flat }}$ velocity function.

Whether the W50/2 velocity function is compatible with $\Lambda \mathrm{CDM}$ cosmology becomes a matter of understanding how measures of W50/2 in a large sample of galaxies relate to measures of $\mathrm{V}_{\text {flat }}$ in galaxies with extended HI discs and regular rotation curves.

When matching galaxies to DM halos by mass, the problem lies with understanding observational measurements of $\mathrm{V}_{\text {rot }}$ rather than a shortfall in the number of galaxies that are "too big to fail". This may require that the halos have DM cores, rather than cusps. Yet we already have significant evidence that many dwarf disc galaxies having slowly rising rotation curves, possibly indicative of cored DM profiles.

Various proposals for explaining the existence of cores have been proposed, both within the $\Lambda$ CDM paradigm (e.g. Navarro et al. 1996, Read et al. 2006, Governato et al. 2010, Pontzen \& Governato 2012), or by taking an alternative form of DM (e.g. Zavala et al. 2009, Vogelsberger et al.|2012, Schneider et al.|2014). In an accompanying paper (Brook \& Di Cintio 2015 submitted), we will compare how galaxy cusps and cores are reflected in the 
velocity function, and then proceed to highlight how different core formation mechanisms may leave signatures in the detailed shape of the velocity function.

According to our analysis, $\mathrm{V}_{\text {rot }}$ is not measured at $\mathrm{R}_{\max }$ in dwarf galaxies. We provide relations between $R_{H I}$ and $R_{\text {eff }}$ of observed galaxies, and $\mathrm{R}_{\mathrm{vir}}$ of their matched $\mathrm{DM}$ halos, and showed that dwarfs are confined to more central regions of their matched DM halos than more massive galaxies. This explains why rotation curves of dwarf galaxies are generally rising at the last measured point. As one does not reach $R_{\max }$ the measured $V_{\text {rot }}$ is dependent on the extent of the HI gas, as well as its detailed structure. Scatter begins to emerge in the various BTFRs for low mass galaxies, and the different measures of $\mathrm{V}_{\text {rot }}$ become increasingly different, leading to different velocity functions.

We performed several consistency checks between different data sets from the literature. In the appendix, we have taken this further, redoing our analysis using the recent data from Bradford et al. (2015), who measured the HI line-widths of a large sample of dwarf galaxies selected from the SDSS catalog. The results, and in particular the large effect of different measures of $V_{\text {rot }}$ on the velocity function, are unchanged from the analysis in the paper.

Our study highlights that if the velocity function is to be used as a probe of cosmological paradigm, we need to be precise in relating measures of $\mathrm{V}_{\text {rot }}$ with the $\mathrm{V}_{\text {max }}$ of DM halos. Not all measures of $V_{\text {rot }}$ are equal and not all BTFRs have the same slope, particularly in low max galaxies. In a forthcoming paper, we will explore various forms of the BTFR within hydrodynamical, cosmological simulations, looking at how the different measures of the BTFR can be reconciled within a $\Lambda \mathrm{CDM}$ context.

\section{ACKNOWLEDGEMENTS}

CB thanks the MICINN (Spain) for the financial support through the MINECO grant AYA2012-31101 and the Ramon y Cajal program. He further thanks the DARK cosmology centre for their kind hospitality.

\section{REFERENCES}

Aumer M., White S. D. M., Naab T., Scannapieco C., 2013, MNRAS, 434, 3142

Begum A., Chengalur J. N., Karachentsev I. D., Sharina M. E., Kaisin S. S., 2008, MNRAS, 386, 1667

Bell E. F., McIntosh D. H., Katz N., Weinberg M. D., 2003, ApJL, 585, L117

Benson A. J., Frenk C. S., Lacey C. G., Baugh C. M., Cole S., 2002, MNRAS, 333, 177

Bradford J. D., Geha M. C., Blanton M. R., 2015, ArXiv e-prints Brook C. B., Di Cintio A., Knebe A., Gottlöber S., Hoffman Y., Yepes G., Garrison-Kimmel S., 2014, ApJ, 784, L14

Brook C. B., Stinson G., Gibson B. K., Roškar R., Wadsley J., Quinn T., 2012, MNRAS, 419, 771

Bullock J. S., Kravtsov A. V., Weinberg D. H., 2000, ApJ, 539, 517

Doyle M. T. et al., 2005, MNRAS, 361, 34

Geha M., Blanton M. R., Masjedi M., West A. A., 2006, ApJ, 653, 240

Governato F. et al., 2010, Nature, 463, 203

Guo Q., White S., Li C., Boylan-Kolchin M., 2010, MNRAS, 404, 1111
Gurovich S., Freeman K., Jerjen H., Staveley-Smith L., Puerari I., 2010, AJ, 140, 663

Haynes M. P. et al., 2011, AJ, 142, 170

Karachentsev I. D., Makarov D. I., Kaisina E. I., 2013, AJ, 145, 101

Klypin A., Karachentsev I., Makarov D., Nasonova O., 2014, ArXiv e-prints

Klypin A. A., Trujillo-Gomez S., Primack J., 2011, ApJ, 740, 102

Koribalski B. S. et al., 2004, AJ, 128, 16

Kravtsov A. V., 2013, ApJ, 764, L31

McGaugh S. S., 2005, ApJ, 632, 859

McGaugh S. S., 2012, AJ, 143, 40

Moster B. P., Somerville R. S., Maulbetsch C., van den Bosch

F. C., Macciò A. V., Naab T., Oser L., 2010, ApJ, 710, 903

Navarro J. F., Eke V. R., Frenk C. S., 1996, MNRAS, 283, L72

Papastergis E., Cattaneo A., Huang S., Giovanelli R., Haynes M. P., 2012, ApJ, 759, 138

Papastergis E., Giovanelli R., Haynes M. P., Shankar F., 2015, A\&A, 574, A113

Papastergis E., Martin A. M., Giovanelli R., Haynes M. P., 2011, ApJ, 739, 38

Peeples M. S., Shankar F., 2011, MNRAS, 417, 2962

Pontzen A., Governato F., 2012, MNRAS, 421, 3464

Read J. I., Wilkinson M. I., Evans N. W., Gilmore G., Kleyna J. T., 2006, MNRAS, 367, 387

Schneider A., Anderhalden D., Macciò A. V., Diemand J., 2014, MNRAS, 441, L6

Shankar F., Lapi A., Salucci P., De Zotti G., Danese L., 2006, ApJ, 643,14

Somerville R. S., 2002, ApJ, 572, L23

Trachternach C., de Blok W. J. G., McGaugh S. S., van der Hulst J. M., Dettmar R.-J., 2009, A\&A, 505, 577

Trujillo-Gomez S., Klypin A., Primack J., Romanowsky A. J., 2011, ApJ, 742, 16

Vogelsberger M., Zavala J., Loeb A., 2012, MNRAS, 423, 3740

Zavala J., Jing Y. P., Faltenbacher A., Yepes G., Hoffman Y., Gottlöber S., Catinella B., 2009, ApJ, 700, 1779

Zwaan M. A., Meyer M. J., Staveley-Smith L., 2010, MNRAS, 403, 1969

\section{APPENDIX A: DIFFERENT DATA SETS}

We have emphasised in our paper the effect of the different measures of the BTFR on the velocity function. Our analysis adopts a set of empirical relations between $\mathrm{M}_{\text {halo }}, \mathrm{M}_{*}, \mathrm{M}_{\text {gas }}$ and $\mathrm{V}_{\text {rot }}$, using data from several different studies. Here, we repeat our analysis but starting with a different set of empirical relations between $\mathrm{M}_{\text {halo }}, \mathrm{M}_{*}, \mathrm{M}_{\text {gas }}$ and $\mathrm{V}_{\text {rot }}$, using the results of the recent study of Bradford et al. (2015). who study HI line-widths of a sample of isolated dwarf galaxies selected from the Sloan Digital Sky Survey. We show that our main conclusions are not altered.

\section{A1 Different baryonic abundance matching}

Our starting point is the empirical $\mathrm{M}_{*}-\mathrm{M}_{\text {halo }}$ relation of Moster et al. (2010), shown as a black line in figure A1, which is slightly flatter at the low mass end than the Guo et al. (2010) relation used in the paper. We then use the double power law relation between $\mathbf{M}_{*}$ and $\mathrm{M}_{\text {gas }}$ from Bradford et al. (2015), which results in the $\mathbf{M}_{\mathrm{b}}$ $\mathrm{M}_{\text {halo }}$ relation shown as the red line in figure $\mathrm{A} 1$ 


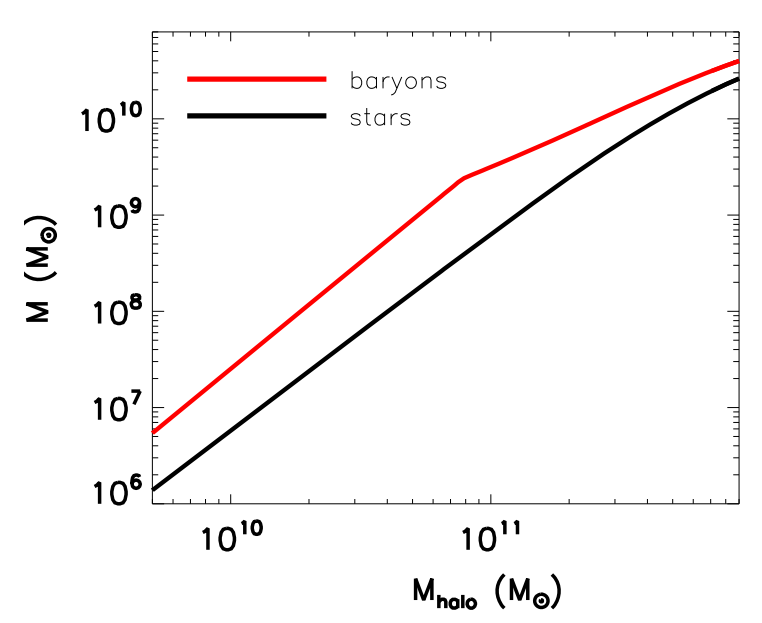

Figure A1. The abundance matching relation adopted in the appendix, $\mathbf{M}_{\mathrm{b}}$ $\mathrm{M}_{\text {halo }}$ (red line) and $\mathrm{M}_{*}-\mathrm{M}_{\text {halo }}$ (black line).

\section{A2 Reconciling BTFRs from a new data set}

Bradford et al. (2015) derive a BTFR using W20/2,

$$
\log _{10} \mathrm{~W}_{20} / 2=-0.668+0.277 \log _{10} \mathrm{M}_{\mathrm{b}}
$$

where there is no correction made for dispersion. This BTFR is shown as a black line in Figure A2 and is is very similar to the BTFR from McGaugh (2012), shown as a blue line. We also show, as a red line, the $\mathrm{V}_{50}$ BTFR from this paper, i.e. equation 4 . The dashed red line adjusts this $\mathrm{V}_{50}$ BTFR to a W20/2 BTFR using equation 5 after also adjusting $\mathrm{V}_{50}$ to $\mathrm{W} 50 / 2$ by including the $8 \mathrm{~km} \mathrm{~s}^{-1}$ velocity dispersion. The resultant relation is very close to the $\mathrm{V}_{20}$ relation of Bradford et al. (2015).

Bradford et al. (2015) find a typical difference between W50 and $\mathrm{W} 20$ is $\sim 25 \mathrm{~km} \mathrm{~s}^{-1}$ (see also Koribalski et al. 2004). We therefore use this value to also transform the Bradford et al. (2015) BTFR (black line) to a W50 BTFR, shown as the dashed black line. The adjusted BTFR is very similar to our adopted W50 BTFR, equation 4 This gives us significant confidence in our analysis, and in particular in equations equation $4 \& 5$ from our paper, which are the key results which are driving the significant differences in the velocity function for different measures of $\mathrm{V}_{\text {rot }}$.

\section{A3 The effect of BTFRs on the Velocity Function}

Starting this time from the abundance matching by mass shown in Figure A1 we show in Figure A3 the effect of the different BTFRs on the velocity function. The yellow line shows the $\mathrm{V}_{\text {max }}$ velocity function of DM only simulations. Using the W50/2 BTFR, equation 4 , is shown as the red line. The W20/2 BTFR, derived using equation 5 is shown as a red dashed line. Results of assuming the BTFR of Bradford (2015), i.e. equation A1 are shown as the black solid line, with the adjustment of W50=W $20-25 \mathrm{~km} \mathrm{~s}^{-1}$ shown as the black dashed line.

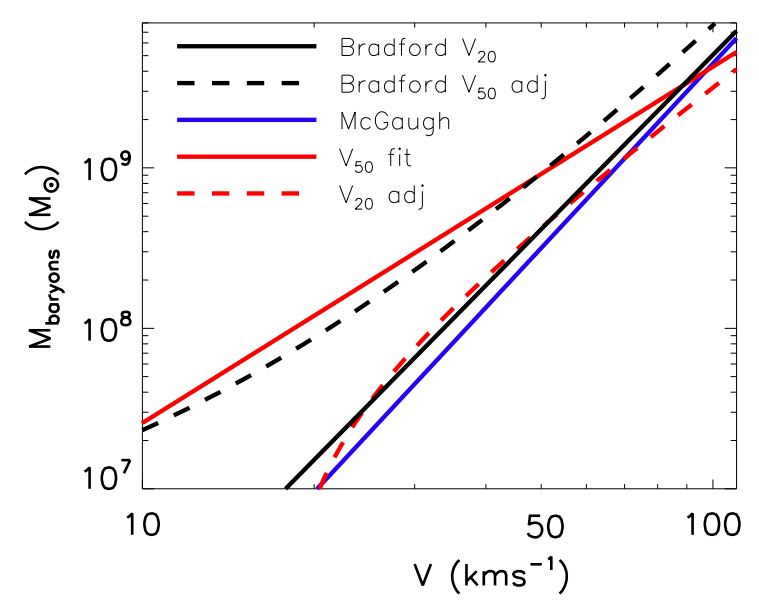

Figure A2. The baryonic Tully-Fisher relation (BTFR) for the data set, with a fit to galaxies. Also shown are the BTFRs of McGaugh 2012, derived using $\mathrm{V}_{\text {flat }}$.

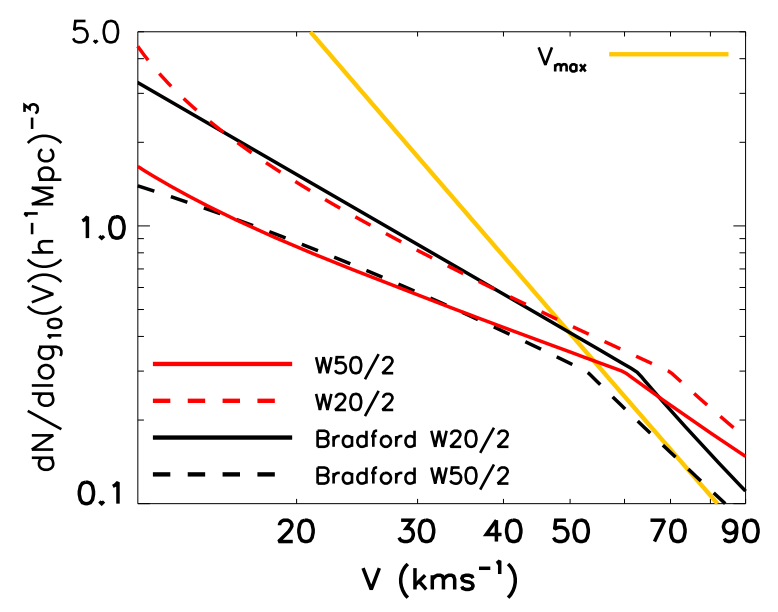

Figure A3. The yellow line shows the $\mathrm{V}_{\max }$ velocity function of DM only simulations. We then show the velocity function predicted by $\Lambda$ CDM cosmology when making different assumptions about the BTFR, having matched observed baryon masses to DM halo masses. Using the W50/2 BTFR, equation 4 is shown as the red line. The W20/2 BTFR, derived using equation 5 is shown as a red dashed line. Results of assuming the BTFR of Bradford (2015), i.e. equation A1 are shown as the black solid line, with the adjustment of $\mathrm{W} 50=\mathrm{W} 20-25 \mathrm{~km} \mathrm{~s}^{-1}$ shown as the black dashed lines. 